\title{
Das MCU bei Kindern. Dosiswerte im klinischen Alltag im Hinblick auf die stark gesenkten diagnostischen Referenzwerte
}

\author{
Dose Area Product of Pediatric VCUG with Regard to the Strongly Lowered \\ German Diagnostic Reference Levels
}

Autoren

Institute
M. Born ${ }^{1}$, L. Spiller ${ }^{1}$, H. Bachour ${ }^{2}$, A. Heydweiller², I. Franke ${ }^{3}$

Radiologische Klinik - Kinderradiologie, Universität Bonn

2 Chirurgische Klinik, Universität Bonn

3 Kinderklinik, Universität Bonn

\author{
Key words \\ - bladder \\ ureter \\ - fluoroscopy \\ technical aspects \\ - diagnostic reference level \\ dose area product
}

\section{Zusammenfassung \\ $\nabla$}

Ziel: Vergleich der im klinischen Alttag bei MCUUntersuchungen von Kindern auftretenden Flächendosisprodukte mit den drastisch gesenkten Referenzwerten, die auf einer mutmaßlich nicht repräsentativen Datenselektion beruhen.

Material und Methoden: 413 konsekutive MCUUntersuchungen bei Kindern und Jugendlichen wurden analysiert.

Ergebnisse: Der Mittelwert aller Untersuchungen lag mit 0,97 $\mathrm{dGycm}^{2}$ unter dem niedrigsten Referenzwert, der für Säuglinge gilt. In 12 Fällen $(5,6 \%)$ wurde der Referenzwert überschritten. Schlussfolgerung: Bei Nutzung der zur Verfügung stehenden Maßnahmen zum Strahlenschutz lassen sich auch im klinischen Alltag die um z.T. $80 \%$ gesenkten Referenzwerte für das MCU einhalten, obwohl die Referenzwerte aus einer vermutlich nicht repräsentativen Datenmenge stammen.

\section{Abstract \\ $\nabla$}

Purpose: To compare the dose area products of pediatric VCUG in daily practice with the dramatically reduced official German diagnostic reference levels, which are based on selected data.

Materials and Methods: 413 consecutive pediatric VCUG examinations were analyzed.

Results: The mean dose area product of all examinations was $0.97 \mathrm{dGycm}^{2}$. This is below the lowest reference level that is valid for neonates. In 12 cases $(5.6 \%)$ the achieved dose area product was higher than the corresponding reference level.

Conclusion: Using the available techniques for radiation protection, it is possible in the daily routine to meet the official diagnostic reference levels for children, which have been reduced by up to $80 \%$, even though these levels are based on a selected, possibly non-representative data set. eingereicht 18.3.2012

akzeptiert $\quad 12.10 .2012$

Bibliografie

Dol http://dx.doi.org/

10.1055/s-0032-1325609

Online-Publikation: 15.11.2012

Fortschr Röntgenstr 2013; 185 :

262-267 @ Georg Thieme

Verlag KG Stuttgart · New York . ISSN 1438-9029

\section{Korrespondenzadresse} Dr. Markus Born

Radiologische Klinik Kinderradiologie, Universität Bonn

Adenauerallee 119

53113 Bonn

Tel.: ++ 49/228/28733225

Fax: $++49 / 228 / 28733566$

mark.born@ukb.uni-bonn.de

\section{Einleitung}

$\nabla$

Dem Strahlenschutz kommt im Kindesalter eine besonders große Bedeutung zu. Dies wurde erst kürzlich in einer Übersichtsarbeit von Alzen und Benz-Bohm erneut dargelegt [1] und spiegelt sich in der Tatsache wider, dass auch aktuelle Studien sich weiterhin mit der Dosisbestimmung und -minimierung bei Röntgenaufnahmen von Kindern beschäftigen [2-5]. Ein hohes Potenzial zur Übertragung von Strahlendosen haben insbesondere Durchleuchtungsuntersuchungen aufgrund der sehr variablen Untersuchungsdauer, die von der Kooperationsfähigkeit und somit dem Alter des zu untersuchenden Kindes mitbestimmt wird. Daher ist eine regelmäßige Erfassung der bei den einschlägigen radiologischen Untersuchungen von Kindern auftretenden Dosisflächenprodukte (DFP) besonders wichtig. Sie dient nicht nur zur Qualitätskontrolle des einzelnen Untersuchers oder Instituts, sondern auch zur Festlegung der offiziellen Referenzwerte. Im Jahr 2010 hat das Bundesamt für Strahlenschutz die geltenden Referenzwerte für radiologische Untersuchungen anhand der ihm vorliegenden selektionierten DFP-Angaben deutlich nach unten korrigiert [6]. Insbesondere für die Miktionszysurethrografie (MCU) wurden die Referenzwerte für das Dosisflächenprodukt stark gesenkt, in Abhängigkeit der Altersgruppe der untersuchten Kinder um 75-83\%. Die dieser Anpassung der Referenzwerte zugrunde liegenden Daten sind die den ärztlichen Stellen im Rahmen der Qualitätssicherung bekannt gewordenen DFP-Werte einer größeren Zahl ausgewählter Untersuchungen, die allerdings nicht zwingend eine repräsentative Stichprobe aller MCU-Untersuchungen an Kindern darstellen. 
Ziel der Arbeit war es, das gesamte mit einem handelsüblichen Durchleuchtungsgerät im klinischen Alltag in einer kinderradiologischen Abteilung auftretende Spektrum von DFP-Werten bei MCU-Untersuchungen von Kindern zu erfassen und mit den geltenden neuen Referenzwerten zu vergleichen.

\section{Patienten und Methoden \\ $\nabla$}

Es wurden retrospektiv alle MCU-Untersuchungen ausgewertet, die durch die beiden zur Stammbesetzung einer kinderradiologischen Abteilung gehörenden Kollegen in den letzten 5 1⁄2 Jahren durchgeführt wurden (Juli 2006 - Dezember 2011). Erfasst wurden die Untersuchungen, die mit der Fragestellung nach vesicoureteralen Refluxen durchgeführt wurden. Nicht erfasst wurden: 1. Untersuchungen, die im Rahmen der Abklärung bestimmter Fehlbildungen (Analatresien, Vaginalatresien mit Kloakenfehlbildung, u.a.) vom üblichen Untersuchungsgang abweichen und z. B. zur Suche einer möglichen Fistel-Fistel-Verbindung mit der Darstellung des Enddarms kombiniert werden; 2. verständlicherweise die Refluxprüfungen, die sonografisch durchgeführt wurden (Miktionsurosonografie $=$ MUS) und 3. solche Untersuchungen, die durch Kollegen durchgeführt wurden, die nur vertretungsweise oder kurzfristig in der Abteilung eingesetzt waren.

Alle Untersuchungen wurden an der abteilungseigenen digitalen Durchleuchtungseinheit (Easy Diagnost - DSI, Philips) mit gittergesteuerter Pulsierung in „Last-Image-hold“-Technik durchgeführt, bei der die unter DL erzielten Monitorbilder digital in den Bildspeicher übernommen werden und auf die Anfertigung von Röntgenaufnahmen weitgehend verzichtet wird. Alle Untersuchungen wurden mit einem Zusatzvorfilter von $1 \mathrm{~mm}$ Al und $0,2 \mathrm{~mm} \mathrm{Cu}$, ohne Streustrahlenraster und mit einer Pulsrate von 3 Bildern pro Sekunde durchgeführt. Auf eine konsequente Einblendung wurde geachtet. Das verwendete Bildwandlersystem besteht aus einem BV mit den zur Verfügung stehenden Bildwandlerformaten $17 \mathrm{~cm}, 25 \mathrm{~cm}$ sowie $38 \mathrm{~cm}$. Die BildwandlerEingangsdosisleistung beim $25 \mathrm{~cm}$ Eingangsfeld liegt bei der verwendeten Pulsrate bei $0,18 \mu \mathrm{Gy} / \mathrm{s}$. Das Dosisflächenprodukt wird hierbei errechnet. Bei Säuglingen wurde die „Kinder-“/,Baby“Taste aktiviert, ein Modus, der garantiert, dass bei einem kleinen Durchleuchtungsvolumen die durch die automatische Dosisleistungsregelung eingestellte Röhrenspannung nicht unter $60 \mathrm{kV}$ sinkt. Bei Säuglingen, die die Harnblase bereits bei geringer Füllung entleerten, wurde die Füllung 1- bis 2-mal wiederholt (sogenanntes zyklisches MCU). Die Untersuchungen erfolgten bei wachen, nicht sedierten oder narkotisierten Kindern.

Insgesamt wurden 413 Untersuchungen bei 379 Kindern ausgewertet, hiervon 227 Mädchen und 152 Jungen. Das Alter der Kinder zum Zeitpunkt der Untersuchung betrug im Mittel 2,6 Jahre (1. Lebenstag bis 17,7 Jahre), 7 Kinder waren älter als 12 Jahre. Eine Darstellung der Altersverteilung liefert@ Abb. 1. Von den 413 Untersuchungen waren 338 Erstuntersuchungen und 75 Wiederholungsuntersuchungen bei vorbestehendem Reflux zur Beurteilung einer Maturation oder zur Therapiekontrolle nach antirefluxiver Harnleiterplastik oder Ureterostiumunterspritzung. Nicht in allen Fällen einer Kontrolluntersuchung war hierbei auch die Erstuntersuchung in unserer Klinik durchgeführt worden. In einem kleinen Teil der Fälle wurde im Verlauf eine 2. Kontrolluntersuchung durchgeführt.

Von den 338 Erstuntersuchungen wurden 215 aufgrund eines erstmaligen oder rezidivierenden Harnwegsinfekts durchgeführt, 123 Untersuchungen aufgrund von sonografischen Auf-

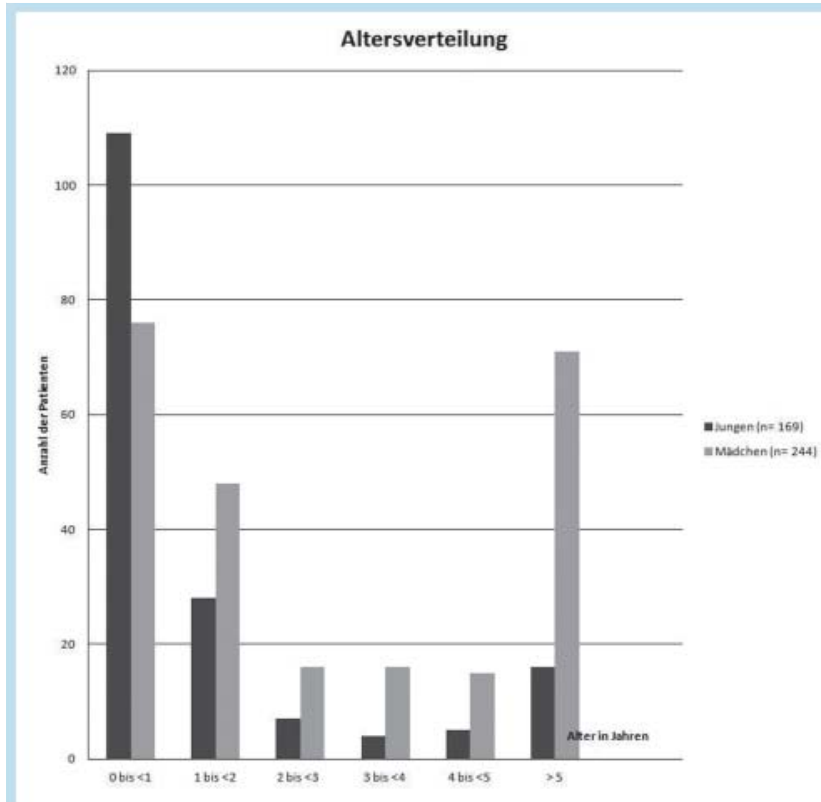

Abb. 1 Übersicht über die Altersverteilung der untersuchten Kinder zu den jeweiligen Untersuchungszeitpunkten.

fälligkeiten (operationspflichtige Subpelvinstenose, einseitiger Megaureter, V.a. Harnröhrenklappe u. a.).

\section{Ergebnisse \\ $\nabla$}

Die mittlere Durchleuchtungszeit betrug 33,6s vergleichbar zu den Angaben in der Literatur [7 - 9]. Das mittlere Dosisflächenprodukt aller Untersuchungen lag bei $0,97 \mathrm{dGycm}^{2}(0,1-$ $11,1 \mathrm{dGycm}^{2}$ ) mit einer Standardabweichung von 1,34 dGycm² Eine detaillierte Übersicht über alle aufgetretenen Dosisflächenprodukte im Vergleich zu den aktuellen Referenzwerten ist in - Abb. 2 zu finden, eine Darstellung der mittleren DFP-Werte in den 4 Altersgruppen, zu denen das Bundesamt für Strahlenschutz Referenzwerte herausgegeben hat, sowie über die Referenzwerte aus den Jahren 2003 und 2010 liefert $\bullet$ Abb. 3, hierbei wurden in der Gruppe „Neugeborene“ Säuglinge bis zu einem Alter von 3 Monaten zusammengefasst. In $\bullet$ Abb. 4 sind in Form eines ,Boxplots' Angaben zur Verteilung der aufgetretenen Dosisflächenprodukte in den verschiedenen Altersgruppen und der Häufigkeit der Überschreitung der Referenzwerte zusammengefasst. Die in den $4 \mathrm{Al}-$ tersgruppen aufgetretenen Dosisflächenprodukte lagen im Mittel bei $25 \%$ bis knapp $55 \%$ des jeweiligen Referenzwerts (40,1\%/ $25,0 \% / 54,6 \%$ bzw. 29,2\% für die Altersgruppen Neugeborene/8 12 Monate/3 - 7 Jahre bzw. 8 - 12 Jahre). Der Mittelwert des Dosisflächenprodukts aller 413 Untersuchungen lag mit $0,97 \mathrm{dGycm}^{2}$ knapp unter dem niedrigsten Referenzwert von 1,0 dGycm² $=$ $10 \mathrm{cGycm}^{2}$, der für Neugeborene gilt. Die Referenzdosiswerte wurden bei 12 von 216 Untersuchungen von den Kindern überschritten, deren Alter in einer der 4 vom Bundesamt für Strahlenschutz genannten Altersklassen lag ( $\bullet$ Tab. 1). Dies entspricht 5,6\% der Untersuchungen. Es handelt sich hierbei um 7 Jungen und 5 Mädchen. Drei der Kinder waren aus der Altersgruppe der Säuglinge, 8 aus der Altersgruppe der 3- bis 7-Jährigen und ein Kind aus der Altersgruppe der 8- bis 12-Jährigen. Die mittlere Durchleuchtungszeit lag bei diesen 12 Kindern mit 78 s deutlich höher als die mittlere Durchleuchtungszeit aller Kinder mit knapp 34 s. Folgen- 


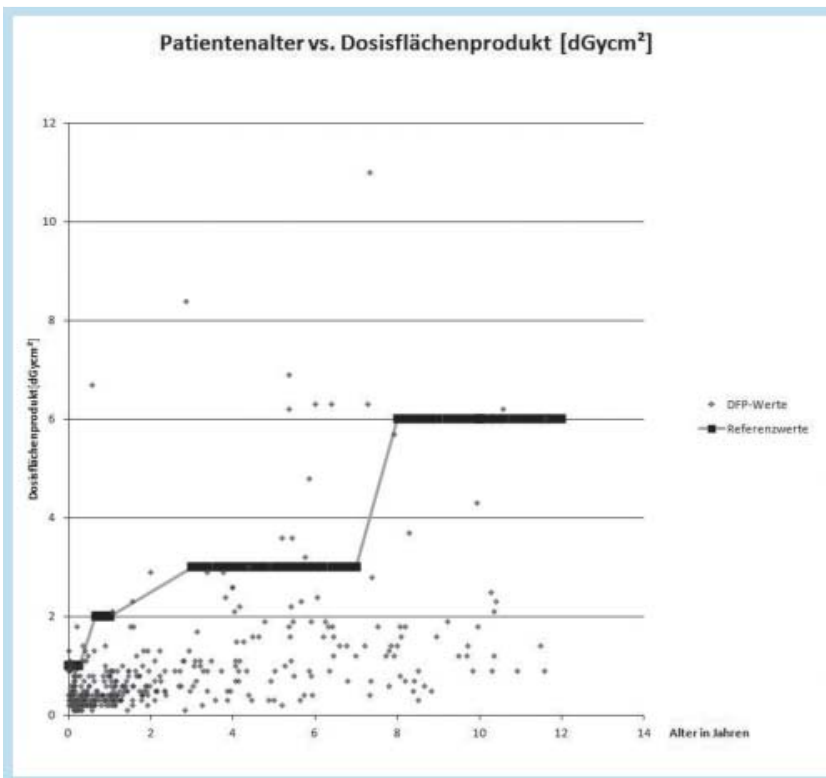

Abb. 2 Dosenflächenprodukte aller Untersuchungen. Markiert sind die neuen Referenzwerte (2010) für die 4 vorgegebenen Altersklassen durch horizontale schwarze Linien.

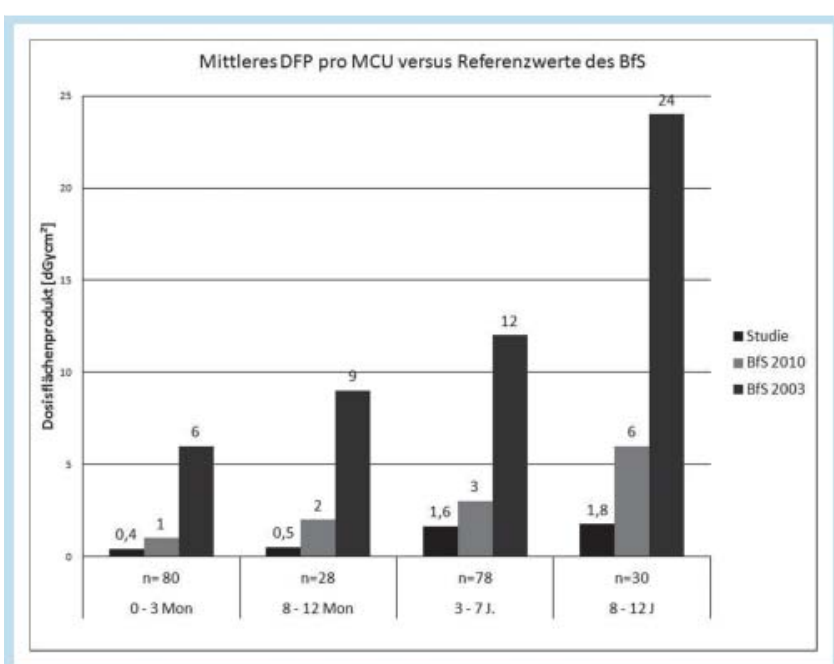

Abb. 3 Übersicht über die mittleren Dosisflächenprodukte in den 4 Altersgruppen (schwarz, jeweils links) im Vergleich zu den Referenzwerten aus den Jahren 2003 (dunkelgrau, jeweils rechts) und 2010 (hellgrau, jeweils mittig).

de Auffälligkeiten wurden im Befundbericht der MCU-Untersuchungen dieser Kinder beschrieben: 6 der Kinder konnten oder wollten lange Zeit kein Wasser lassen, in 3 Fällen wurde nach sehr langem Zuwarten letztlich ein Töpfchen auf dem Untersuchungstisch montiert, der hierzu vertikal gestellt werden musste. Ein Junge mit Urethralklappe vermochte wiederholt nur äußerst kleine Urinportionen zu lassen, sodass es zunächst nicht möglich war, die kontrastierte Urethra zu dokumentieren. Bei einem Jungen mit Doppelnierenanlage, Ureterozele und hochgradigem Reflux in die untere Nierenanlage kam es zunächst immer wieder zu Unterbrechungen der Miktion unmittelbar nach Miktionsbeginn. Bei einem Jungen mit Lipomeningomyelozele und eingeschränkter

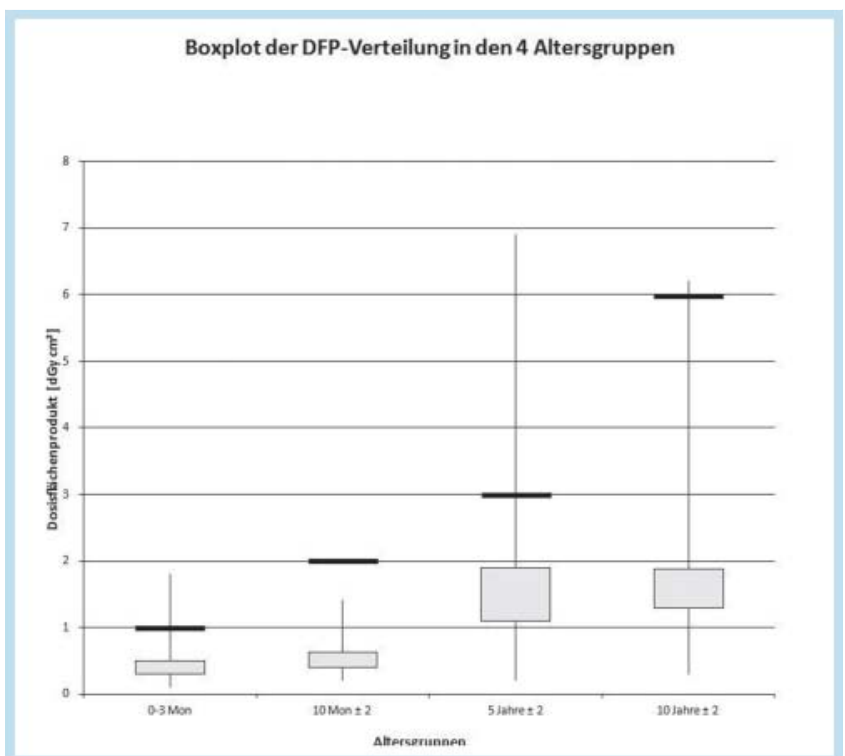

Abb. 4 Darstellung der DFP-Verteilung in den 4 Altersgruppen als Boxplot: Die Kästchen entsprechen jeweils den mittleren $50 \%$ der Untersuchungsergebnisse, die senkrechten Linien dem Bereich der im oberen und unteren Viertel aufgetretenen DFP-Werte. Die Referenzwerte sind durch horizontale schwarze Balken markiert.

Tab. 1 Mittlere Dosisflächenprodukte der einzelnen Altersgruppen und Häufigkeit der Überschreitung der Referenzwerte.

\begin{tabular}{|c|c|c|c|c|}
\hline & \multicolumn{4}{|c|}{ Altersgruppe } \\
\hline & $\begin{array}{l}\text { Neu- } \\
\text { geborene }\end{array}$ & $\begin{array}{l}10 \text { Mon } \\
\pm 2\end{array}$ & $\begin{array}{l}5 \text { Jahre } \\
\pm 2\end{array}$ & $\begin{array}{l}10 \text { Jahre } \\
\pm 2\end{array}$ \\
\hline $\begin{array}{l}\text { Anzahl der Unter- } \\
\text { suchungen }\end{array}$ & 80 & 28 & 78 & 30 \\
\hline Referenzdosis (dGycm²) & 1,0 & 2,0 & 3,0 & 6,0 \\
\hline mittlere DFP (dGycm²) & 0,4 & 0,5 & 1,6 & 1,8 \\
\hline Minimum & 0,1 & 0,2 & 0,2 & 0,3 \\
\hline Maximum & 1,8 & 1,4 & 6,9 & 11 \\
\hline SD & 0,3 & 0,3 & 1,4 & 1,5 \\
\hline \multicolumn{5}{|l|}{$\begin{array}{l}\text { Überschreitung der } \\
\text { Referenzdosis }\end{array}$} \\
\hline Anzahl der Fälle & 3 & 0 & 8 & 1 \\
\hline Anzahl in \% & $3,75 \%$ & $0,00 \%$ & $10,26 \%$ & $3,33 \%$ \\
\hline
\end{tabular}

Blasenkontrolle dauerte es längere Zeit, bis nach zunächst nur winzigen Urinportionen zumindest ein kurzer Harnstrahl zustande kam. Für ein Mädchen war im Befund der Zuweiser eine Detrusor-Sphinkter Dyskoordination beschrieben mit abgeflachtem, mehrgipfligem Profil im Flow EMG (Uroflowelektromyografie). Bei einem Mädchen war die Kooperationsfähigkeit stark eingeschränkt wegen eines Dysmorphiesyndroms mit Intelligenzminderung bei gleichzeitig bestehendem Anfallsleiden (ICD 10 E34.3 und G40.0) und bei einem Jungen waren wegen fehlender Angaben im Untersuchungsbericht die Gründe für das erhöhte DFP retrospektiv nicht mehr eruierbar. 


\section{Diskussion}

\section{(}

Der beste Strahlenschutz ist die vollständige Vermeidung ionisierender Strahlung, sodass der Miktionsurosonografie (MUS) eine zunehmende Bedeutung als Alternative zur klassischen MCU zukommt. Die MUS ist inzwischen gut evaluiert [10-13] und wird in den Leitlinien der Gesellschaft für Pädiatrische Radiologie (GPR) (S1-Leitlinie, Stand 3/2011) zur Abklärung einer Harntransportstörung als Alternative zur MCU-Untersuchung bei Mädchen mit einseitiger Dilatation der ableitenden Harnwege oder zur Refluxprüfung bei gesichertem erstem fieberhaftem Harnwegsinfekt empfohlen $[14,15]$. Eine MUS ist jedoch aus verschiedenen Gründen nicht in jeder Abteilung durchführbar. So bedarf es hierzu moderner Schallgeräte mit einem speziellen Untersuchungsmodus und einer entsprechenden Expertise auf Seiten der Untersucher. Eine Miktionsurosonografie ist relativ zeitaufwendig und blockiert Abteilungen mit nur einem Schallgerät unter Umständen den sonografischen Arbeitsplatz. Ferner ist bislang nur ein einziges Ultraschallkontrastmittel (Levovist) zur Durchführung einer MUS bei Kindern zugelassen, das jedoch derzeit auf dem Markt nicht verfügbar ist [16]. Die Verwendung anderer Ultraschallkontrastmittel bedeutet somit eine sogenannte „Off-Label“-Anwendung. Das MCU hat aus diesen und weiteren Gründen bislang immer noch eine weite Verbreitung und einen akzeptierten Stellenwert als Basisuntersuchung zur Refluxprüfung bei Kindern, der sich auch in den Leitlinien der kinderradiologischen Fachgesellschaft widerspiegelt [17].

Da die Refluxprüfung in der Kinderradiologie eine durch die $\mathrm{Zu}-$ weiser häufig angeforderte Untersuchung ist, ist es um so wichtiger, sie in den Fällen, in denen sie durch eine MCU durchgeführt wird, mit möglichst geringer Röntgendosis durchzuführen.

Für die bei einer Untersuchung auftretende Durchleuchtungszeit gibt es hierbei keine Referenzwerte. Die in unserer Studie aufgetretenen Durchleuchtungszeiten von im Mittel knapp 34 s (Maximum 3,1 min, Standardabweichung 19,8 s) lagen im Bereich der in der Literatur zu findenden Angaben. So geben Persinakis et al. für 118 MCU-Untersuchungen bei pädiatrischen Patienten eine mittlere Durchleuchtungszeit von $44 \mathrm{~s}$ für Mädchen und $55 \mathrm{~s}$ für Jungen an [7]. Darling et al. berichten über eine mittlere Durchleuchtungszeit von $47 \mathrm{~s}$ bei 10594 Untersuchungen mit einer deutlichen Reduktion im Verlauf auf $29 \mathrm{~s}$ in den letzten beiden Jahren des Untersuchungszeitraums. Ward et. al ermittelten bei 145 Kindern im Alter von 3 Tagen bis 8 Jahren, unabhängig vom Körperdurchmesser der Kinder, eine mittlere Durchleuchtungszeit von 2 min [9]. Da die Durchleuchtungszeit neben anderen Parametern das Dosisflächenprodukt unmittelbar beeinflusst, wird sie indirekt auch über das DFP erfasst.

Nach Ward lässt sich durch Verwendung einer gepulsten digitalen Durchleuchtungseinheit eine DFP-Reduktion um 80 - 90\% erreichen [9]. Bei Speicherung der digitalen Durchleuchtungsbilder ist hierbei die routinemäßige Anfertigung von zusätzlichen Röntgenzielaufnahmen nicht erforderlich. So berichten Feffermann et al. sowie Bazopoulos et al. über eine hohe Übereinstimmung bzgl. der Beurteilung des Vorhandenseins vesicoureteraler Refluxe zwischen LIH-Aufnahmen und zusätzlich angefertigten Röntgenzielaufnahmen von 94,7 bzw. 97,2\% der untersuchten NierenUreter-Einheiten, bei allerdings relativ kleinen untersuchten Fallzahlen von 65 bzw. 57 MCU-Untersuchungen [18, 19]. Bazopoulos et al. konnten eine Reduktion der Hautdosis um über $50 \%$ durch Verzicht auf zusätzliche Röntgenzielaufnahmen erreichen [19]. In den Empfehlungen der Strahlenschutzkommission zur diagnostischen Bildgebung beim Kind aus dem Jahr 2006 heißt es: „In der Kinderradiologie muss bei Durchleuchtungsuntersuchungen mit gepulster Durchleuchtung gearbeitet werden.“ und „Die Dokumentation über das Speicherbild (last-hold-image) ist in aller Regel ausreichend.“ [20].

Die für das MCU vom Bundesamt für Strahlenschutz veröffentlichten Referenzwerte wurden im Juni 2010 drastisch gesenkt, je nach Altersgruppe um 75-83\% der bislang geltenden Werte. Grundlage der Korrekturen ist die 75. Perzentile der Daten, die den ärztlichen Stellen bei der Wahrnehmung ihrer Aufgaben zur Qualitätskontrolle bekannt gemacht wurden. Hierbei werden die in Deutschland auftretenden DFP-Werte jedoch keineswegs systematisch erfasst, sondern es wird von jeder Abteilung, in der solche Untersuchungen durchgeführt werden, lediglich eine kleine Stichprobe von in der Regel 10 Untersuchungen in größeren Abständen auf die Einhaltung der entsprechenden Vorschriften überprüft. Hierbei fordern nicht alle ärztliche Stellen gezielt bestimmte Untersuchungen an, in vielen Fällen bleibt den zu überprüfenden (kinder-)radiologischen Abteilungen und Praxiseinrichtungen die Auswahl der Untersuchungen überlassen, die der ärztlichen Stelle bekannt gemacht werden. Es ist zu erwarten, dass die überprüften Institutionen die Tendenz aufweisen werden, vorwiegend die Daten gut gelungener Untersuchungen mit eher niedrigen erzielten DFP-Werten mitzuteilen, um mögliche Nachfragen und eventuelle Rechtfertigungen zu vermeiden. Institute mit niedrigen Untersuchungszahlen brauchen z.T. keine DFP-Werte anzuzeigen. Gerade in solchen Instituten könnten jedoch aufgrund geringerer Erfahrung potenziell höhere DFP-Werte auftreten. Es ist also davon auszugehen, dass die den ärztlichen Stellen bekannt gemachten DFP-Werte keine repräsentative Stichprobe aller auftretenden Dosisflächenprodukte darstellen. Da zudem nur 4 Altersgruppen abgefragt werden, in die dtl. weniger als $50 \%$ aller Untersuchungen fallen, wie weiter unten erörtert wird, wird insgesamt nur ein kleiner, selektionierter Teil der Untersuchungen erfasst.

In der vorliegenden Untersuchung sollte daher überprüft werden, welches Spektrum an DFP-Werten in der täglichen Routine bei der Durchführung eines MCU nach dem aktuellen Stand der Technik tatsächlich auftreten und wie sich diese Werte in Bezug auf die geltenden Referenzwerte verhalten. Insbesondere sollte keine Auswahl von bestimmten DFP-Werten erfolgen, sondern es wurden alle im Untersuchungszeitraum aufgetretenen Werte erfasst, deren Untersuchungen den oben beschriebenen Einschlusskriterien genügten. Dies ist insofern von Bedeutung, als die Dauer von Durchleuchtungsuntersuchungen und somit auch die auftretende Strahlendosis gerade bei der Untersuchung von Kindern stark vom Verhalten und der Kooperationsfähigkeit des einzelnen Patienten abhängt und somit stärkeren Schwankungen unterliegt. Lässt ein Kind beispielsweise bereits bei sehr geringer Harnblasenfüllung das erste Mal Wasser, so ist eine zweite, ggf. sogar eine dritte Füllung mit zusätzlichen kurzen Durchleuchtungszeiten abzuwarten (Zyklisches MCU). Ist das Kind sehr ängstlich und rutscht durch eine unvorhergesehene starke Bewegung plötzlich aus der ,Fixierung', zum Beispiel den haltenden Händen des Untersuchers an den Füßen, so ist eine Korrektur der Position erforderlich, die u.U. eine kurze Durchleuchtung erforderlich macht, damit z. B. die Urethra eines Knaben bei beginnender Miktion sofort im Untersuchungsfeld liegt. Wird der Beginn der Miktion durch ein etwas älteres Kind mehrfach angekündigt, ohne dass sie auch tatsächlich erfolgt, kann dies zu einer Erhöhung der Dosis führen, ebenso wie ein wiederholtes abruptes Unterbrechen der Miktion, wodurch eine Darstellung der kontrastierten Urethra verhindert wird. 
Aufgrund der Selektion der den ärztlichen Stellen bekanntgemachten DFP-Werte einerseits und der zu erwartenden möglichen Schwankungsbreite der auftretenden DFP-Werte andererseits ist es nur schwer abschätzbar, ob tatsächlich $75 \%$ der bundesweit durchgeführten MCU-Untersuchungen bei Kindern den geänderten Referenzwerten genügen, die auf der Basis der 75. Perzentile der gemeldeten Werte erstellt wurden.

Da die auftretenden Dosisflächenprodukte stark von der Körpergröße bzw. dem durchleuchteten Körpervolumen und damit eng korreliert vom Alter eines untersuchten Kindes abhängen, wurden Referenzwerte für 4 Altersgruppen definiert: Neugeborene, 8 - 12 Monate, 3 - 7 Jahre und 8 - 12 Jahre.

In diese Altersklassen fielen 164 der 413 in unserer Studie erfassten Untersuchungen (39,7\%). Da in den ersten 30 Lebenstagen nur relativ selten MCU-Untersuchungen durchgeführt werden, wurden bei den Auswertungen in dieser Arbeit alle Kinder bis zum Alter von 3 Monaten in die Altersklasse Neugeborene aufgenommen, so dass der Anteil der auswertbaren Untersuchungen auf 52,3\% (216 Untersuchungen) angehoben werden konnte.

Die in der vorliegenden Studie erhobenen Daten zeigen, dass in nahezu allen Fällen die Referenzwerte eingehalten wurden. Sogar der Mittelwert aller Untersuchungen lag mit 0,97 dGycm² knapp unterhalb des Referenzwerts für Neugeborene von $1,0 \mathrm{dGycm}^{2}$.

Bei 12 von 216 Untersuchungen (5,6\%) von Kindern im Alter der Referenzaltersgruppen wurde der Referenzwert überschritten. Nach dem Bundesamt für Strahlenschutz [6] stellen die Referenzwerte obere Richtwerte dar, die im Mittel nicht überschritten werden sollen, keine festen Obergrenzen, die nicht überschritten werden dürfen. Auch die Internationale Strahlenschutzkommission hält unter anderem wegen der möglichen Variabilitäten der Untersuchungsbedingungen vorgegebene feste Obergrenzen des DFP für eine Untersuchung nicht als geeignetes Mittel zum Strahlenschutz [21]. Entsprechend bestätigen die Ergebnisse der vorliegenden Studie, dass es bei komplexeren Befunden (Doppelniere mit Ureterozele, Urethralklappe, Meningozele mit gestörter Harnblasenfunktion) sowie bei eingeschränkter Kooperationsfähigkeit der Kinder in wenigen Fällen zu einem DFP oberhalb der Referenzwerte kommen kann. Am häufigsten trat dies in der Altersgruppe der 3- bis 7-jährigen Kinder auf, die beginnen, einen eigenen Willen zu entwickeln, wodurch auch die Kooperationsbereitschaft eingeschränkt sein kann.

Es ist somit durchaus zulässig, dass einzelne Untersuchungen den Referenzwert überschreiten. Eine Vorschrift dazu, in wie viel Prozent der Einzelfälle ein Referenzwert höchstens überschritten werden darf, existiert nicht. Zum einen führt das Bundesamt für Strahlenschutz (BfS) aus, dass Referenzwerte als Perzentile einer beobachteten Verteilung der mittleren Patientendosen verschiedener Anwender (z.B. die 75. Perzentile) festgelegt sind [6], sodass man rückfolgern könnte, dass auch beim einzelnen Untersucher in nicht mehr als $25 \%$ der Fälle die Referenzwerte überschritten werden sollte. Zum anderen wird jedoch durch das BfS betont, wie oben zitiert, dass lediglich die Mittelwerte der auftretenden Dosisflächenprodukte die Referenzwerte nicht überschreiten sollen. Dies ist eine relativ schwache Vorgabe, da bei schiefen Verteilungen durchaus mehr als die Hälfte der Fälle den Mittelwert überschreiten kann, sodass Konstellationen denkbar sind, in denen die Anforderung des BfS erfüllt ist, obwohl in mehr als der Hälfte der Fälle der Referenzwert überschritten wird. Noch schwächer sind die Vorgaben durch die Röntgenverordnung (§17a, Absatz1), nach der erst eine beständige, ungerechtfertigte Überschreitung der Do- sisreferenzwerte den zuständigen Landesbehörden zu melden ist [22].

Es erscheint zunächst erstaunlich, dass die in der vorliegenden Studie berichteten DFP-Werte deutlich unter den stark gesenkten Referenzwerten lagen, obwohl davon auszugehen ist, dass die Referenzwerte auf der Basis von Untersuchungen erstellt wurden, deren DFP-Werte im unteren Bereich der auftretenden Dosisflächenprodukte lagen. Eine mögliche Erklärung könnte daran liegen, dass die den ärztlichen Stellen gemeldeten Werte, die an älteren, nicht digitalen Durchleuchtungsanlagen erzielt wurden, ebenfalls in die Erstellung der Referenzwerte eingeflossen sind. Auf Anfrage wurde den Autoren von Seiten des Bundesamts für Strahlenschutz mitgeteilt, dass bei der Erstellung der Referenzwerte nicht zwischen den Angaben von DFP-Werten an konventionellen Durchleuchtungsanlagen und an digitalen Durchleuchtungsanlagen unterschieden wurde. Für den Gesetzgeber ist es eine schwierige Aufgabe einheitliche Referenzwerte für technisch so unterschiedliche Gerätetypen festzulegen. Problematisch wäre es, Gerätetypen, die die gesetzlichen Bestimmungen einhalten, für bestimmte Untersuchungen auszuschließen. Da sich jedoch mit der digitalen Technik das Dosisflächenprodukt gegenüber der konventionellen Technik erheblich senken lässt, wie oben ausgeführt [9], und zudem zu erwarten ist, dass die Zahl der im Einsatz befindlichen Altgeräte insbesondere auch durch Verschärfungen der gesetzlichen Bedingungen deutlich abnimmt (so sind seit dem 1.1.2012 Durchleuchtungsuntersuchungen nur noch an Geräten mit dosissparenden Kennlinien zulässig), sind weitere zukünftige Überarbeitungen der Referenzwerte wünschenswert, die das sich ändernde Gerätespektrum widerspiegeln. Hierzu bieten die hier vorgestellten Daten einen Anhaltswert, reichen jedoch bei Weitem nicht aus, da sie lediglich an einem einzigen Zentrum erhoben wurden und daher nicht repräsentativ sein können.

\section{Beurteilung}

$\nabla$

Die vorliegenden Ergebnisse zeigen, dass sich bei Verwendung der zur Verfügung stehenden Möglichkeiten zum Strahlenschutz (erfahrender Untersucher, digitale Durchleuchtung mit niedriger Pulsfrequenz, 'Last-Image-hold'-Modus mit weitgehendem Verzicht auf die Anfertigung von zusätzlichen Aufnahmen, Zusatzfilterung, Verzicht auf Streustrahlenraster, Verwendung einer in der SV-RL geforderten geringen Bildverstärkereingangsdosisleistung und konsequente Einblendung) bei Kindern in der täglichen Routine niedrige DFP-Werte bei der MCU-Untersuchung erzielen lassen, sodass sich nicht nur für ausgewählte Fälle, sondern für das Gesamtkollektiv - auch bei schwierigen Untersuchungsbedingungen - die stark gesenkten Referenzwerte des Bundesamts für Strahlenschutz in aller Regel einhalten und in den meisten Fällen deutlich unterschreiten lassen. Die im Jahr 2010 gesenkten Dosisreferenzwerte sind jedoch weiterhin optimierbar.

\section{Literatur}

1 Alzen G, Benz-Bohm G. Radiation protection in pediatric radiology. Dtsch Arztbl Int 2011; 108: 407-414

2 Hess $R$, Neitzel U. Optimizing Image Quality and Dose for Digital Radiography of Distal Pediatric Extremities Using the Contrast-to-Noise Ratio. Fortschr Röntgenstr 2012; 184: 643-649

3 Schneider K, Seidenbusch MC. Zur Strahlenexposition von Kindern in der pädiatrischen Radiologie Teil 8: Strahlendosen beim thorakoabdo- 
minalen Babygramm und bei der Abdomenaufnahme Neugeborener und Säuglinge. Fortschr Röntgenstr 2010; 182: 479-492

4 Hirsch W, Sorge I. Ein Literatur-Review zur wissenschaftlichen Präsenz der deutschen Kinderradiologie in Publikationen der Jahre 2009 2011. Fortschr Röntgenstr 2011; 183: 1012-1015

5 Grieser T, Baldauf AQ Ludwig K. Radiation dose reduction in scoliosis patients: low-dose full-spine radiography with digital flat panel detector and image stitching system. Fortschr Röntgenstr 2011; 183: $645-649$

6 Noßke D. Bekanntmachung der aktualisierten diagnostischen Referenzwerte für diagnostische und interventionelle Röntgenuntersuchungen. Bundesamt für Strahlenschutz 22.6.2010.

7 Perisinakis K, Raissaki M, Damilakis J et al. Fluoroscopy-controlled voiding cystourethrography in infants and children: are the radiation risks trivial? Eur Radiol 2006; 16: 846 - 851

8 Darling S, Sammer M, Chapman $T$ et al. Physician documentation of fluoroscopy time in voiding cystourethrography reports correlates with lower fluoroscopy times: a surrogate marker of patient radiation exposure. Am J Roentgenol 2011; 196: 777-780

9 Ward VL, Strauss KJ, Barnewolt CE et al. Pediatric radiation exposure and effective dose reduction during voiding cystourethrography. Radiology 2008; 249: 1002 - 1009

10 Vassiou K, Vlychou M, Moisidou R et al. Contrast-enhanced sonographic detection of vesicoureteral reflux in children: comparison with voiding cystourethrography. Fortschr Röntgenstr 2004; 176: 1453-1457

11 Darge K. Voiding urosonography with US contrast agent for the diagnosis of vesicoureteric reflux in children: an update. Pediatr Radiol 2010; 40: 956-962

12 Darge $K$. Voiding urosonography with ultrasound contrast agents for the diagnosis of vesicoureteric reflux in children. I. Procedure. Pediatr Radiol 2008; 38: 40-53
13 Darge $K$. Voiding urosonography with ultrasound contrast agents for the diagnosis of vesicoureteric reflux in children. II. Procedure. Pediatr Radiol 2008; 38: 54-63

14 Mentzel HJ, Stöver B, Moritz J et al. Leitlinie „Harntransportstörung bei Kindern - Bildgebende Diagnostik“, Registernr: 064/006. www.awmf. com

15 Mentzel HJ, Stöver B, Moritz J et al. Leitlinie „Harnwegsinfekt bei Kindern - Bildgebende Diagnostik“, Registernr: 064/007. www.awmf.com

16 Piscaglia F, Nolsoe C, Dietrich CF et al. The EFSUMB Guidelines and Recommendations on the Clinical Practice of Contrast Enhanced Ultrasound (CEUS): Update 2011 on non-hepatic applications. Ultraschall in Med 2012; 32: $33-59$

17 Dacher JN. Dagnostic Procedures Excluding MRI, Nuclear Medicine, and Video-Urodynamics. In: Baert AL, Knauth M, Sartor K (eds) Pediatric Uroradiology Berlin Heidelberg: Springer Verlag; 2010, 1-15

18 Fefferman NR, Sabach AS, Rivera $R$ et al. The efficacy of digital fluoroscopic image capture in the evaluation of vesicoureteral reflux in children. Pediatr Radiol 2009; 39: 1179-1187

19 Bazopoulos EV, Prassopoulos PK, Damilakis JE et al. A comparison between digital fluoroscopic hard copies and $105-\mathrm{mm}$ spot films in evaluating vesicoureteric reflux in children. Pediatr Radiol 1998; 28 : $162-166$

20 Strahlenschutzkommission. Bildgebende Diagnostik beim Kind Strahlenschutz, Rechtfertigung und Effektivität - Empfehlung der Strahlenschutzkommission. www.ssk.de. 12.7.2006

21 Internationale Strahlenschutzkommission. Strahlenschutz und Sicherheit in der Medizin; ICRP-Veröffentlichung 73; BFS-SCHR-1999. Bremerhaven: Wirtschaftsverlag/Verlag für neue Wissenschaft

22 Sonnek C, Bauer B. Die neue Röntgenverordnung. Verordnung über den Schutz vor Schäden durch Röntgenstrahlen (Röntgenverordnung RöV) vom 8. Januar 1987 (BGBI. IS. 114), in der Fassung der Bekanntmachung vom 30. April 2003 (BGBl. IS. 604). Berlin: H. Hoffmann GmbH Verlag; 2003 